\title{
Lithiases Enclavees Dans L'uretre: Une Pathologie Pas Aussi Rare A L'hopital Somine Dolo De Mopti
}

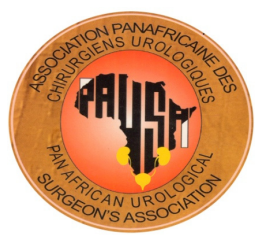

\author{
Cissé $D^{1}$, Berthé $\mathrm{HJG}^{2}$, Diarra $A^{5}$, Coulibaly $\mathrm{MT}^{3}$, Diallo $\mathrm{MS}^{4}$, Kassogué $A^{4}$, Thiam ${ }^{6}$, Traoré $A^{1}$, \\ Koné $M^{1}$, Guindo O. $^{1}$
}

${ }^{1}$ Hôpital Sominé DOLO de Mopti, ${ }^{2} \mathrm{CHU}$ du Point G Bamako, ${ }^{3} \mathrm{CHU}$ Gabriel TOURE Bamako, ${ }^{4} \mathrm{CHU}$ Kati

${ }^{5}$ Direction Régionale de la Santé de Mopti.

\section{Résumé}

Introduction: L'urètre n'est pas une localisation fréquente des lithiases. Beaucoup d'études rapportent des cas cliniques ou des séries limitées. L'objectif de cette étude était de montrer la fréquence de cette affection dans notre pratique.

Patients et méthode: Il s'agissait d'une étude rétrospective descriptive portant sur tous les patients pris en charge pour lithiase urétrale dans notre structure entre le 1er Juin 2012 et le 30 Juin 2016.

Résultats: 106 cas de lithiases urétrales ont été diagnostiqués et traités. Cette localisation a représenté $14,54 \%$ de l'ensemble des urolithiases $(n=729)$ et 3,13\% des interventions urologiques durant la période $(n=3384)$. L'âge moyen des patients était de 14, 24 ans avec des extremes de 1 et 68 ans. Le sex ratio était de 7, 15 en faveur des hommes. Le motif de consultation était la retention aiguë d'urines dans $66,98 \%$ des cas $(n=71)$. Le calcul était palpable dans $83,02 \%(n=88)$ des cas. Le traitement a consisté à une cystolithotomie après réfoulement du calcul dans la vessie dans $74,53 \%(\mathrm{n}=79)$ des cas, à une extraction instrumentale dans $21,70 \%(\mathrm{n}=23)$ des cas et à une urétrolithotomie dans $03,77 \%(\mathrm{n}=4)$ des cas. L'urètre était permeable à l'ablation du calcul dans tous les cas.

Conclusion: La lithiase enclave dans l'urètre n'est pas une affection rare dans notre pratique. Elle doit être évoquée devant une retention aiguë d'urines chez l'enfant et l'adolescent.

Mots clés: Lithiase, urètre, retention aiguë d'urines, cystolithotomie.

Rec, u le: 7 August 2019, Accepté le: 8 August 2019

Auteur correspondant: Cissé Dramane, Service de Chirurgie Hôpital Sominé DOLO de Mopti (MALI) BP 139, Email: djennecisse@yahoo.fr

ISSN: 2090-2379, https://afju.journals.ekb.eg

\section{Introduction}

La lithiase urinaire est la présence de concrétion pierreuse sur les voies urinaires. Sa formation, faite de cristallogenèse presque normale, de calculogénèse, de rétention et croissance du calcul; donne une place de plus en plus importante à la plaque de Randall ${ }^{[1]}$. L'étiologie de la majorité des lithiases est inconnue. Une origine génétique est évoquée car la présence de cas familiaux augmente le risque de lithiase par $2,5^{[2]}$. La prévalence de la pathologie lithiasique est plus élevée chez les jumeaux homozygotes que chez les jumeaux hétérozygotes ${ }^{[3]}$. La prévalence de la lithiase urinaire est variable dans le monde, entre 7 et $13 \%$ en Amérique du Nord, 5 et $9 \%$ en Europe et 1 à $5 \%$ en Asie Sud-est avec des zones d'endémie lithiasique comme le Sud-est de la Turquie ${ }^{[4]}$. La localisation sur le tractus urinaire est aussi variable, plus fréquent sur le haut appareil urinaire dans les pays développés et sur le bas appareil urinaire dans les pays en développement ${ }^{[5]}$. La localisation urétrale est rare et estimée de $0,3 \%^{[6]}$ à $2 \%^{[7]}$ de l'ensemble des lithiases. Cette lithiase urétrale peut être primaire, c'està-dire constituée dans l'urètre à la faveur d'une sténose urétrale ou d'un diverticule, ou secondaire par migration d'un calcul rénal ou vésical ${ }^{[8]}$. La localisation urétrale est plus fréquente chez les enfants particulièrement dans les pays en développement à cause de la prévalence élevée de la lithiase vésicale ${ }^{[9]}$. L'objectif de cette étude est de déterminer la place de la lithiase urétrale dans la pathologie lithiasique et de décrire sa prise en charge.

\section{Patients et méthode}

Il s'agissait d'une étude rétrospective descriptive portant sur les patients traités pour lithiase urétrale entre le

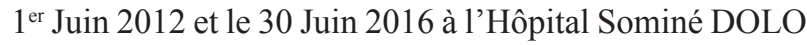
de Mopti. Les patients ont été admis en urgence ou vu en consultation de routine. Chaque patient a bénéficié d'un examen physique et d'examen d'imagerie. Le traitement a 
consisté à une cystolithotomie pour la majorité des lithiases, exception faite des lithiases de la fossette naviculaire et des lithiases affleurant le méat urétral chez l'homme et chez la femme. Cette cystolithotomie a été faite après refoulement de la lithiase dans la vessie à l'aide de la pression hydraulique $\mathrm{du}$ contenu en sérum physiologique d'une seringue de $20 \mathrm{ml}$ appliquée étanchement sur le méat urétral. Dans les cas où l'instillation du sérum physiologique à travers l'urètre était inefficace, le refoulement a été délicatement réalisé à vessie ouverte, à l'aide d'un béniqué de calibre convenable suffisamment lubrifié. Dans les cas où aucune mobilisation n'a été possible, nous avons procédé à une urétrolithotomie. Ces interventions ont été réalisées sous anesthésie générale chez les patients de moins de 16 ans et sous rachi anesthésie à partir de 16 ans. Les lithiases de la fossette naviculaire où celles affleurant le méat urétral ont bénéficié d'une extraction instrumentale après anesthésie locale.

Une fiche d'enquête conçue comportait l'identité du malade, les antécédents, le motif de consultation, les données de l'examen clinique, les résultats des examens complémentaires, le traitement et le suivi du malade. Cette fiche a été remplie à partir du dossier médical, du registre de compte rendu opératoire. 131 dossiers de lithiases urétrales ont été colligés. Nous avons exclu les cas de lithiases urétrales expulsés spontanément avant l'admission à l'hôpital, les cas de lithiases se projetant dans l'urètre postérieur qui étaient dans la vessie à la cystotomie et les cas pris en charge sans la preuve d'imagerie. L'exploitation informatique des données a été réalisée à l'aide du logiciel Epi Info version 3.3.2. Notre analyse a porté sur la fréquence des variables.

\section{Résultats}

$\mathrm{Au}$ total, 106 cas de lithiase urétrale ont été retenus sur un total de 729 cas de lithiase urinaire soit un taux de $14,54 \%$. Les interventions d'ablation de lithiase urétrale ont constitué 3,13\% de l'ensemble des activités chirurgicales du service $(n=3384)$. Sur le tableau 1 est représentée l'incidence annuelle des lithiases urétrales.

Tableau 1: Incidence des lithiases urétrales

\begin{tabular}{lcc}
\hline ANNEE & FREQUENCE & $\%$ \\
\hline 2012 & 17 & 16,04 \\
2013 & 22 & 20,75 \\
2014 & 19 & 17,92 \\
2015 & 21 & 19,82 \\
2016 & 27 & 25,47 \\
TOTAL & 106 & 100,00 \\
\hline
\end{tabular}

L'âge moyen des patients a été de 14,24 ans avec des extrêmes de 1 et 68 ans. Le sexe ratio de prédominance masculine était de 7,15. Sur le tableau 2 est représentée la répartition des patients en fonction de l'âge et du sexe.
Tableau 2: Repartition en Fonction de L'age et du Sexe

\begin{tabular}{lccc}
\hline $\begin{array}{l}\text { SEXE } \\
\text { TRANCHE }\end{array}$ & MASCULIN & FEMININ & TOTAL \\
D'AGE & & & \\
\hline $0-5$ ans & 30 & 1 & 30 \\
$6-10$ ans & 39 & 4 & 43 \\
$11-15$ ans & 9 & 3 & 12 \\
$16-25$ ans & 6 & 3 & 9 \\
60 ans et plus & 9 & 2 & 11 \\
TOTAL & 93 & 13 & 106 \\
\hline
\end{tabular}

Le motif de consultation était une rétention aiguë d'urines dans $66,98 \%$ des cas, une dysurie et des prurits péniens dans $14,15 \%$ et $10,38 \%$ des cas respectivement. Les antécédents pathologiques recensés étaient les douleurs lombo-abdominales, la dysurie, la douleur pelvienne dans $13,21 \%, 11,32 \%, 6,60 \%$ respectivement. Les différents motifs de consultation et les antécédents pathologiques sont illustrés dans les tableaux 3 et 4 .

Tableau 3: Motif de Consultation

\begin{tabular}{lcc}
\hline $\begin{array}{l}\text { MOTIFS DE } \\
\text { CONSULTATIONS }\end{array}$ & FREQUENCE & $\%$ \\
\hline $\begin{array}{l}\text { Rétention aigüe } \\
\text { d'urines }\end{array}$ & 71 & 66,98 \\
Dysurie & 15 & 14,15 \\
Prurit pénien & 11 & 10,38 \\
Pyurie & 4 & 03,78 \\
Incontinence & 3 & 02,83 \\
urinaire & & \\
Perception de masse & 2 & 01,88 \\
TOTAL & 106 & 100,00 \\
\hline
\end{tabular}

Tableau 4: antécédent Urologiques

\begin{tabular}{lcc}
\hline ANTECEDANTS & FREQUENCE & $\%$ \\
\hline $\begin{array}{l}\text { Douleurs abdomino- } \\
\text { lombaires }\end{array}$ & 14 & 13,21 \\
Dysurie & 12 & 11,32 \\
Douleurs & 7 & 06,60 \\
hypogastriques & 5 & \\
Hématurie & 4 & 04,73 \\
Emission de calcul & 2 & 03,77 \\
Lithotomie & 62 & 01,88 \\
Aucun & 106 & 58,49 \\
TOTAL & & 100,00 \\
\hline
\end{tabular}


A l'examen physique, la lithiase était palpable dans $83,03 \%(\mathrm{n}=88)$ des cas. Un globe vésical était présent dans $71,70 \%(n=76), 9,43 \%(n=10)$ des patients présentaient une tuméfaction pénienne, $5,67 \%(\mathrm{n}=6)$ une hypertrophie prostatique d'allure bénigne au toucher rectal et deux patientes une cystocèle du 2ème degré.

Les examens d'imagerie réalisés ont été la radiographie de l'abdomen sans préparation dans $83,02 \%$ des cas $(\mathrm{n}=88 \%)$ et l'échographie de l'arbre urinaire dans $16,98 \%$ $(\mathrm{n}=18)$ des cas. Les examens biologiques réalisés ont été la calcémie, l'uricémie et la créatinémie. Ils n'ont pas relevé d'anomalie biologique notable. Au terme du bilan, la lithiase était située dans l'urètre antérieur dans 73,59\% $(\mathrm{n}=78)$ des cas, (figure 1) dans l'urètre postérieur dans $14,15 \%(\mathrm{n}=15)$ des cas (figure 2). Elle était dans l'urètre pelvien ou périnéal féminin dans $7,55 \%(\mathrm{n}=8)$ des cas (figure 3 ) et au méat urétral féminin dans $4,41 \%(n=5)$ des cas.

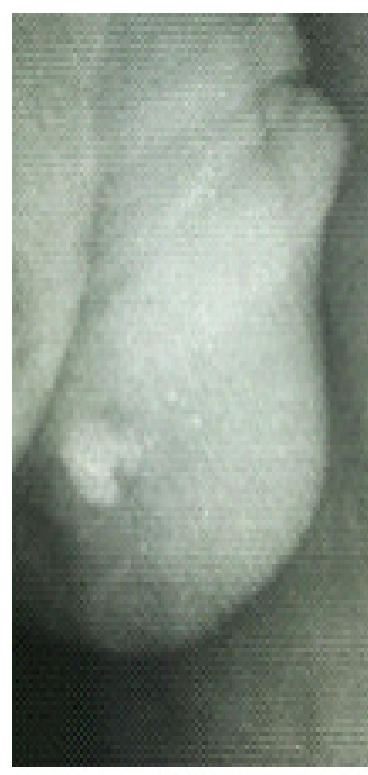

a

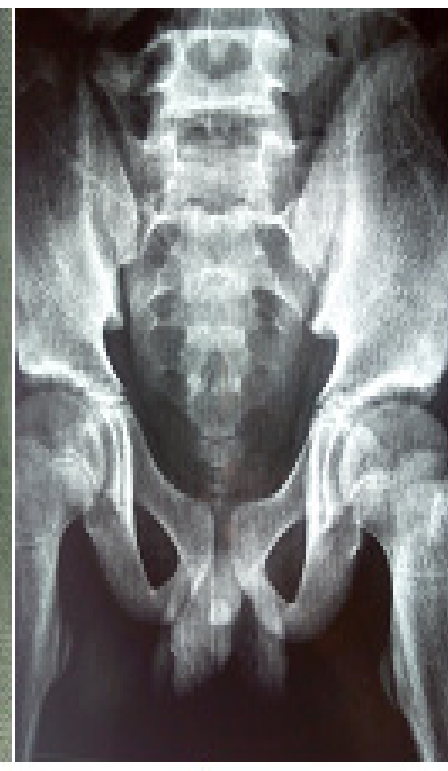

b
Figure 1. a : calcul dans lafossette naviculaire b : cakul medio-pénienne

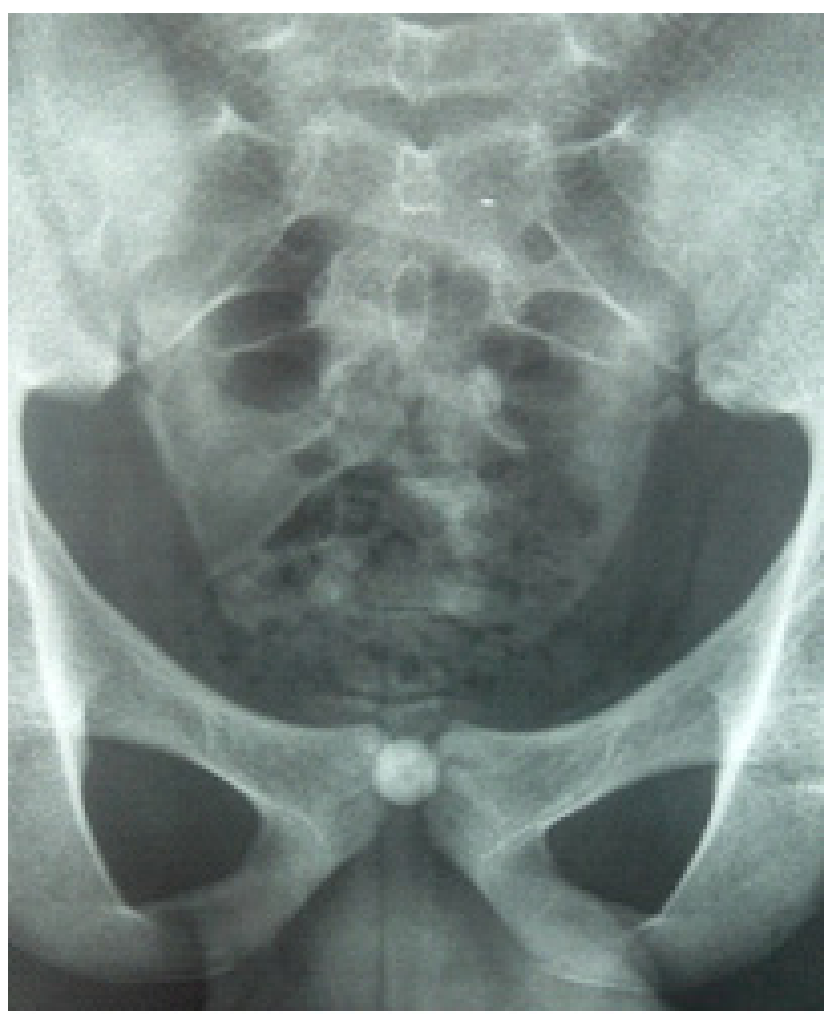

Figure 2. Calcul de l'urẻtre postérieur

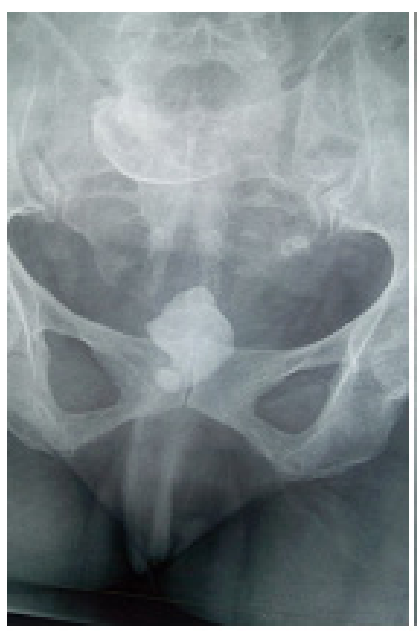

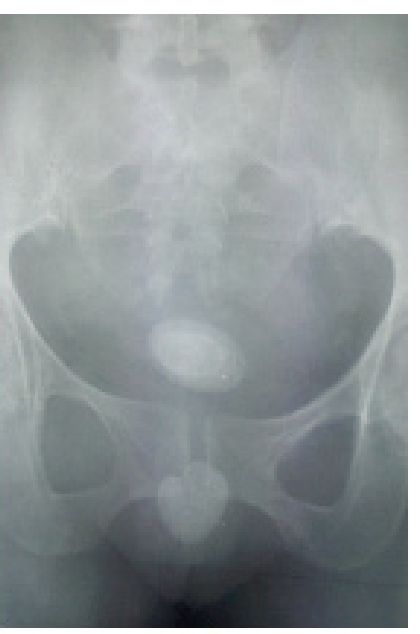

b
Figure 3. Calcul de Purètre féminin.

a: calcul vésical enclavé dansle col.

b : localisation multiple, vésicale et urétrale. 
Des localisations multiples, vésicale, urétérale et calicielle ont représenté $16,98 \% \quad(\mathrm{n}=18)$ des cas (Figure 4).

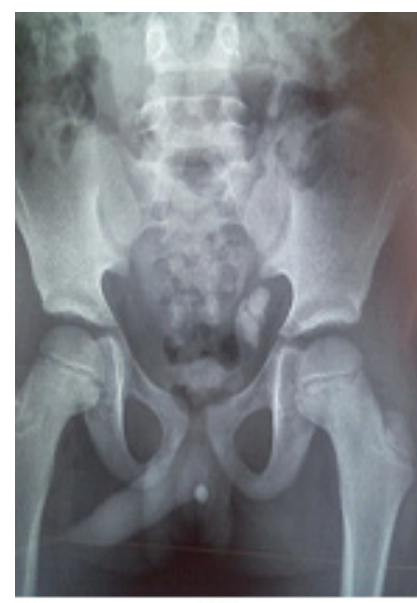

a

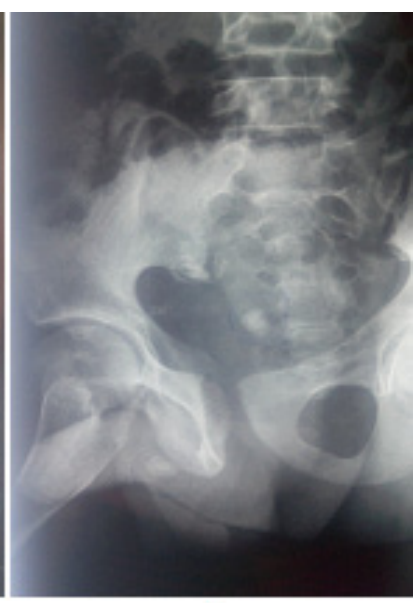

Figure 4. Localisation multiple.

a: urétrale et urétérale gauche

b : urétrale et vésicale

Chez un patient de 24 ans de sexe masculin sans antécédent familial de calcul et sans anomalie biologique, ont été retrouvés un double calcul urétral associé à deux lithiases dans chaque bas uretère et une lithiase calicielle droite (Figure 5).

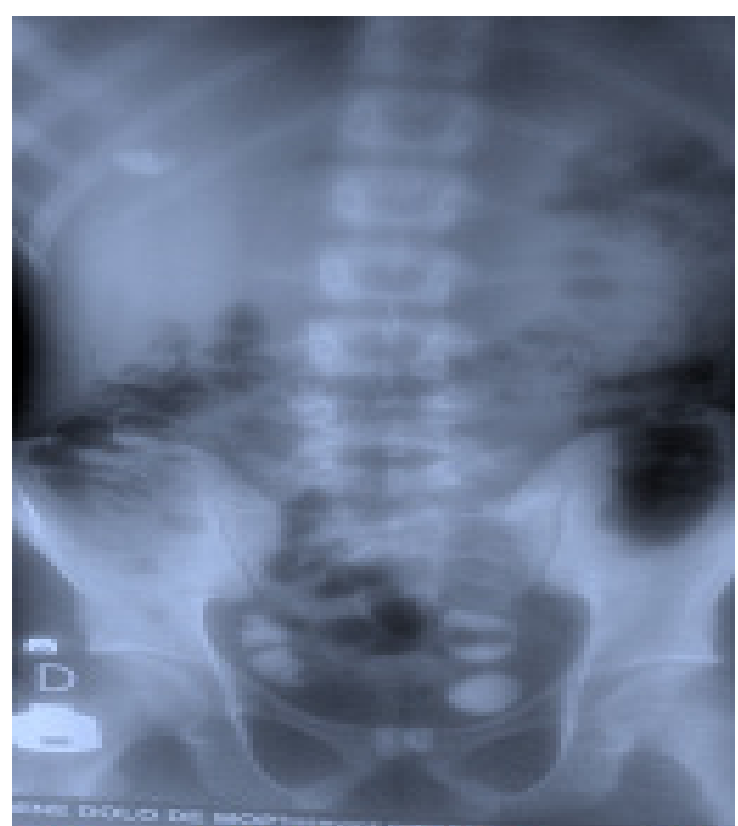

Figure 5 : localisation multiple Double urétral

Double urétérale droite Double ureterale gauche Calicielledroite
Dans les tableaux 5, 6 et 7 sont représentées respectivement, la localisation des calculs, leurs localisations en fonction de l'âge et du sexe et les pathologies associées.

Tableau 5 : Localisation De La Lithiase

\begin{tabular}{lcc}
\hline LOCALISATION & FREQUENCE & $\%$ \\
\hline Urètre pénien & 32 & 30,19 \\
Urètre périnéal & 29 & 27,36 \\
Fossette naviculaire & 17 & 16,04 \\
Urètre postérieur & 15 & 14,15 \\
$\begin{array}{l}\text { Urètre pelvien/ } \\
\text { périnéal féminin }\end{array}$ & 8 & 07,55 \\
Méat urétral féminin & 5 & 04,71 \\
Total & 106 & 100,00 \\
\hline
\end{tabular}


Tableau 6 : Localisation De La Lithiase En Fonction De L'age En Annee Et Du Sexe

\begin{tabular}{|c|c|c|c|c|c|c|}
\hline & $0-5$ & 6- 10 & $11-15$ & $16-25$ & 60 et plus & TOTAL \\
\hline Urètre pénien & 15 & 12 & 1 & 1 & 3 & 32 \\
\hline Urètre périnéal & 8 & 12 & 3 & 2 & 4 & 29 \\
\hline $\begin{array}{l}\text { Urètre } \\
\text { postérieur }\end{array}$ & 4 & 6 & 2 & 1 & 2 & 15 \\
\hline $\begin{array}{l}\text { Fossette } \\
\text { naviculaire/ } \\
\text { méat urétral }\end{array}$ & 3 & 9 & 3 & 2 & 0 & 17 \\
\hline $\begin{array}{l}\text { Urètre périnéal } \\
\text { féminin }\end{array}$ & 1 & 2 & 2 & 2 & 1 & 8 \\
\hline $\begin{array}{l}\text { Méat urétral } \\
\text { féminin }\end{array}$ & 0 & 2 & 1 & 1 & 1 & 5 \\
\hline TOTAL & 31 & 43 & 12 & 9 & 11 & 106 \\
\hline
\end{tabular}

Tableau 7: Repartition En Fonction Des Pathologies Associees

\begin{tabular}{lcc}
\hline PATHOLOGIES ASSOCIEES & FREQUENCE & $\%$ \\
\hline Sténose du méat urétral & 12 & 11,33 \\
Lithiase calicielle & 7 & 06,61 \\
Lithiase vésical & 4 & 03,79 \\
HBP & 4 & 03,79 \\
Lithiase bas uretère & 4 & 03,79 \\
HBP et lithiase vésicale & 2 & 01,88 \\
cystocèle & 2 & 01,88 \\
Lithiase calicielle et urétérale & 1 & 0,9 \\
Aucune & 70 & 66,03 \\
TOTAL & 106 & 100,00 \\
\hline
\end{tabular}

Le traitement a consisté en une cystolithotomie après refoulement de la lithiase dans la vessie dans $64,15 \%(n=68)$ des cas, une extraction instrumentale dans $21,70 \%(n=23)$ des cas, dont $13,21 \%(\mathrm{n}=14)$ après méatotomie (12 cas de sténose du méat urétral et 2 cas de lithiases volumineuses par rapport au méat). L'adénomectomie prostatique transvésicale et l'urétérolithotomie ont été réalisées en plus de la cystolithotomie respectivement dans $5,67 \%(n=6)$ et $4,71 \%(\mathrm{n}=5)$ des cas. L'urétrolithotomie a été réalisée dans 4 cas $(3,77 \%), 2$ cas de lithiase enclavés à l'angle pénoscrotale et 2 cas de lithiase bloqués au niveau de l'urètre bulbaire. Les lithiases calicielles et les cystocèles de 2ème degré n'ont pas fait l'objet de prise en chirurgicale. Les lithotomies ont été faites sous anesthésie générale dans $64,16 \%(n=68)$, rachi-anesthésie dans $18,87 \%(n=20)$ et sous anesthésie locale dans $16,97 \%$ des cas $(n=18)$. Les suites opératoires ont été marquées par 7 cas d'infection pariétale et 3 cas de fistules vésico-cutanées, toutes prises en charge avec succès. Les mictions ont dans l'ensemble été satisfaisantes à la fin du traitement.

\section{Discussion}

La localisation urétrale de la lithiase est rare. Beaucoup de publication retrouvée dans la littérature décrivaient des cas cliniques ou des séries limitées. Quelques articles portant sur plus d'une dizaine de cas, sur une période d'au moins 3 ans ont été retrouvés sur Pubmed. La lithiase urétrale ne représente que $0,3 \%$ de l'ensemble des urolithiases ${ }^{[6]}$. Malgré ce faible pourcentage, elle est rencontrée fréquemment dans les zones endémiques comme l'Asie et le Moyen-Orient particulièrement chez les enfants ${ }^{[10]}$. La lithiase urétrale est retrouvée dans notre étude avec une incidence annuelle moyenne de 21,2 cas, elle a représentée $14,54 \%$ de l'ensemble des urolithiases. Cette incidence élevée est due à la fréquence hospitalière des urolithiases qui ont représenté $21,54 \%$ des interventions urologiques. Aucune étude n'a été faite sur la prévalence des lithiases dans notre localité pour la classer zone endémique, mais le climat chaud et sec avec une température dépassant $40^{\circ} \mathrm{C}$ pendant une bonne période de l'année pourrait favoriser la lithogénèse comme dans les pays endémiques comme l'Arabie Saoudite ${ }^{[11]}$ 
où la prévalence dans la population générale atteint $20 \%$. L'impact d'une température élevée dans la genèse des urolithiases a été aussi démontré chez les travailleurs en milieu surchauffé tel que dans l'industrie de l'acier ${ }^{[2]}$. La différence de prévalence de la lithiase urinaire entre le Sudest et le Nord-Ouest des Etats-Unis serait due en partie à la température ${ }^{[13]}$.

L'âge moyen a été de 14,24 ans avec des extrêmes de 1 et 68 ans. $69,81 \%(n=74)$ des patients avaient moins de 11 ans. VERIT et col. ${ }^{[10]}$ en Turquie, ont rapporté un âge moyen de 26,2 ans, avec 8 patients (53,3\%) âgé de 4 à 10 ans. Notre âge moyen est inférieur à celui de KAMAL et col. ${ }^{[14]}$ en Arabie Saoudite qui ont rapporté une moyenne d'âge de 36 ans avec un seul enfant de 6 ans. L'incidence élevée des lithiases vésicales chez les enfants dans les pays en développement ${ }^{[9]}$ expliquerait la fréquence élevée des lithiases urétrales pédiatriques dans notre région où l'alimentation à base de céréale, principalement le mil serait un facteur favorisant ${ }^{[9]}$. Entre 26 et 59 ans, aucun patient n'a présenté une lithiase urétrale. Le fort débit mictionnel qui va de 35 à $15-20 \mathrm{ml} / \mathrm{S}$ entre 14 à 50 ans, ${ }^{[15]}$ permettant parfois l'émission des calculs pendant la miction pourrait en être l'explication. VERIT et col. ${ }^{[10]}$ n'ont également enregistré aucun cas à la deuxième et troisième décade. Pour KAMAL et col. [14], la prévalence va de 0 à $18 \%$ pour les deuxièmes et troisièmes décades. Ce fort débit mictionnel et la brièveté de l'urètre pourraient être à la base de la rareté de la lithiase urétrale chez la femme. En effet, en 1996 LARKIN $^{[16]}$ dénombrait approximativement 25 cas dans la littérature. Treize cas de lithiase de l'urètre féminin ont été diagnostiqués pendant notre période d'étude dont huit cas chez les moins de 16 ans. Plusieurs études dépassant la dizaine de cas n'ont pas enregistré de cas féminin ${ }^{[10,14,17,18 ~ 19]}$, SHARFI $^{[20]}$ par contre rapporta deux cas féminins sur 36. La lithiase de l'urètre féminin est associée dans la majorité des cas à un diverticule ${ }^{[21,22]}$. Dans l'ensemble de nos cas féminin, aucune anomalie urétrale n'a été retrouvée comme dans le cas clinique de THOMAS ${ }^{[22]}$ renforçant l'hypothèse de lithiase secondaire par migration.

La rétention aiguë d'urines rencontrée dans 66,98\% des cas a été le motif de consultation le plus fréquent. Ce taux est inférieur à ceux de Kamal et col ${ }^{[14]}$, et SALMAN et col. ${ }^{[18]}$ qui ont tous deux rapporté $78 \%$ de rétention aigüe d'urines, mais nettement supérieur à ceux de $\mathrm{SHARFI}^{[20]}$ et VERIT et col. ${ }^{[10]}$ qui ont rapporté respectivement $22,22 \%$ et $46,7 \%$. La rétraction du pénis par le patient lui-même signalé chez tous les patients de SALMAN ${ }^{[18]}$, que nous avons assimilé à un prurit pénien a été retrouvée dans $10,38 \%(\mathrm{n}=11)$ de nos patients. Ce signe est reconnu par la population locale comme un signe de lithiase urinaire. Le calcul était palpable chez $83,2 \%(n=88)$ de nos patients. Un sondage urétral avec une sonde d'aspiration, plus rigide qu'une sonde de Foley, d'un urètre préalablement bien lubrifié et anesthésié à la lidocaïne gel urétral jusqu'au niveau du calcul, permettait d'éliminer une sténose de l'urètre. Cette attitude nous a dispensés de recourir à l'urétro-cystographie rétrograde, un examen invasif et surtout d'amoindrir le coût de la prise en charge chez une population pas toujours financièrement prête dans un contexte d'urgence. Ainsi, l'examen paraclinique réalisé a été la radiographie de l'abdomen sans préparation dans tous les cas où la lithiase était palpable. Cet examen avait surtout pour but de rechercher d'autre localisation lithiasique et a permis d'en trouver dans $16,98 \%$ des cas $(n=18)$. L'échographie de l'arbre urinaire a été réalisée dans les autres cas de symptomatologie urinaire où le calcul n'était pas palpable. Parmi les 93 cas de lithiase urétrale masculine, le calcul était dans l'urètre antérieur dans 78 cas $(83,87 \%)$ et dans l'urètre postérieur dans 15 cas $(16,13 \%)$. AHMED ${ }^{[23]}$ au Niger, HASSAN ${ }^{[24]}$ au Nigéria et SALMAN ${ }^{[18]}$ en Turquie trouvaient également une majorité de localisation au niveau de l'urètre antérieur respectivement 71,42\% (5/7), 74\% (14/19) et 83,33\% $(50 / 60)$. Ce résultat contraste avec ceux de KAMAL ${ }^{[14]}$ et SHARFI ${ }^{[20]}$ qui ont rapporté une majorité de localisation au niveau de l'urètre postérieur respectivement $88 \%$ et $58,82 \%$. En l'absence d'une sténose de l'urètre, la localisation d'un calcul en migration dépend de son volume, de sa forme et surtout de la durée de la rétention aiguë d'urines qu'il engendre. Un patient en rétention aiguë d'urines avec des tentatives multiples de miction pourrait parvenir à faire déplacer le calcul et arrivant parfois jusqu'à l'expulser. Cette expulsion du calcul est quelque fois empêchée par une sténose du méat urétral, retrouvée chez 11,33\% $(\mathrm{n}=12)$ de nos patients, donnant lieu à des calculs enclavés dans la fossette naviculaire ou en partie accouchés par le méat urétral. La sténose du méat urétral peut aussi déterminer une obstruction qui, à la longue pourrait déterminer la lithogénèse. L'obstruction peut provenir d'une hypertrophie bénigne de la prostate, favorisant la formation de lithiase vésicale qui pourrait migrer dans l'urètre par la suite. La lithiase urétrale était associée à une hypertrophie bénigne da la prostate dans quatre cas et à une hypertrophie bénigne de la prostate associée à une lithiase vésicale dans deux cas. Les localisations multiples, vésicale, urétérale et calicielle, retrouvées chez 18 patients (16,98\%) sont en faveur d'une migration de calcul dans l'urètre.

Le traitement des calculs urétraux doit respecter les impératifs d'être le moins invasif et respecter l'intégrité de l'urètre au tant que faire ce peut. D'une manière générale, les calculs de l'urètre postérieur sont refoulés dans la vessie et traités comme une lithiase vésicale et les calculs de l'urètre antérieur bénéficient d'une extraction instrumentale. Cela suppose de disposer d'une instrumentation suffisante comme les forceps endoscopiques, l'endoscopie miniature pour les enfants et un lithotriteur, toutes choses dont nous sommes dépourvues. Hormis les calculs de la fossette naviculaire et du méat urétral, extraits par des pinces sous anesthésie locale par bloc pénien et parfois après incision du méat urétral, presque tous les autres calculs ont été 
refoulés dans la vessie et extraits par cystolithotomie. La pression hydraulique du contenu en sérum physiologique d'une seringue de $20 \mathrm{ml}$ étanchement appliquée sur le méat urétral a permis le refoulement du calcul dans la vessie. Dans certaines situations ou le calcul est peu obstructif, le liquide passe autour du calcul et ne peut le refouler. Nous avons utilisé dans ces cas, une sonde béniqué lubrifiée pour refouler le calcul dans la vessie, aidé en cela par un doigt intravésical appliqué sur le col pour orienter, faciliter l'entrée dans la vessie et éviter ainsi les fausses routes. Ces manœuvres ont été employées pour ne pas réaliser l'urétrolithotomie que nous n'avons pas pu éviter dans 4 cas où les calculs sont restés inamovibles malgré les tentatives de refoulement. Les calculs vésicaux, et urétéraux associés ont été extraits dans le même temps opératoire ainsi que l'adénomectomie prostatique transvésicale. La méthode qui consiste à traire les calculs de l'urètre antérieur ayant donné jusqu'à $78 \%$ de succès pour SHERIF ${ }^{[25]}$ est difficilement réalisable chez les enfants constituant la majorité de notre effectif. Le retard de consultation, certains patients consultant après plusieurs jours de supplice et le risque de gangrène signalé par MICHAEL ${ }^{[26]}$ nous ont confortés dans notre décision de supprimer le temps d'observation dans l'espoir d'obtenir une expulsion spontanée. L'extraction percutanée sus pubienne des calculs de l'urètre postérieur refoulés dans la vessie à la manière d'une néphrolithotomie percutanée prônée par AHMED et col. ${ }^{[19]}$, de part son incision minime et le court temps de cathétérisme vésical, nous a paru fort élégant ; encore faudrait il disposer de matériel pour fragmenter et extraire le calcul.

Une des insuffisances notables de notre étude réside dans le fait que les calculs extraits, par défaut de plateau technique adéquat, n'ont pu être analysé afin de connaître leur composition chimique. La connaissance de ces compositions chimiques pourrait donner en partie une explication à la fréquence des lithiases dans notre milieu.

\section{Conclusion}

La lithiase urétrale est une urgence fréquente dans notre pratique. Elle touche en majorité les enfants et son traitement a été le plus souvent chirurgical. En plus des facteurs favorisant connu comme la chaleur, les transpirations, l'insuffisance d'hydratation, l'alimentation à base de céréale et les maladies diarrhéiques, d'autres études doivent être menées pour rechercher une corrélation entre la fréquence des lithiases et la qualité de l'eau dans notre région.

\section{Intérêts concurrents}

Les auteurs déclarent ne pas avoir de conflits d'intérêts en relation avec cet article.

\section{Ethique}

Le consentement éclairé des parents a été obtenu pour l'utilisation des images et l'exploitation du dossier médical à des fins scientifiques.

\section{Contribution Des Auteurs}

Dr Babacar Sine, Dr Maguette Faye ont activement participé à la rédaction et à la correction de l'article.

Dr Alioune Sarr, Dr Modou Ndiaye, Dr Amath Thiam, Dr Abdoulaye Ndiath, Dr Ndiaga Seck Ndour1, Dr Faddwa Erradja, Dr Aboubacar Traoré, Dr Samba Thiapato Faye et nous avons collaboré ensemble dans la prise en charge du patient, la rédaction et la correction de ce document.

Les professeurs Yaya Sow, Boubacar Fall, Babacar Diao, Alain K. Ndoye, Mamadou Ba sont les responsables d'enseignement dans notre service. Ils ont tous contribué à la rédaction et la correction de cet article.

Financement: Aucun financement

Consentement à la publication: Non applicable.

\section{Références}

1. Daudona M, Traxer O, Lechevallier E, Saussine C. La lithogénèse. Prog Urol (2008) 18, 815-827.

2. Curhan GC, Willett WC, Rimm EB, Stampfer MJ. Family history and risk of Kidney stones. J Am Soc Nephrol 1997; 8:1568-73.

3. Goldfarb DS, Fischer ME, Keich Y, Goldberg J. A twin study of genetic and Dietary influences on nephrolithiasis: a report from the Vietnam Era Twin (VET) Registry. Kidney Int 2005; 67:1053-61.

4. Sorokin I, Mamoulakis C, Miyazawa K, Rodgers A, Talati J, Lotan Y. Epidemiology of stone disease across the world. World J Urol 2017.

5. Daudon M, Bounxouei B, Santa Cruz F, Leite Da Silva $\mathrm{S}$, et al. Composition des calculs observés aujourd'hui dans les pays non industrialisés. Prog Urol, 2004; 14:1151-61.

6. Aegukkatajit S. Reduction of urinary stone in children from north-eastern Thailand. J Med Assoc Thai 82:1230-1233. 
7. Tsze DS, Kessler DO. Rapid evaluation of urinary retention and penile pain using point-of-care ultrasound. Pediatr Emerg Care 2014; 30(8): 580-2.

8. Vashishtha S, Sureka SK, Agarwal S, Srivastava A, Prabhakaran S, Kapoor R, et al. Urethral stricture and stone: their coexistence and management. Urol $\mathrm{J}$ 2014; 11(1): 1204-10.

9. Menon M, Parulkar BG, Drach GW et al. Urinary lithiasis. Etiology, diagnosis and medical management. In Walsh PC, Retik AB, Vaughan ED, Wein AJ eds, Campbell's Urology.

10. Verit A, Savas M, Ciftci H, et al. Outcomes of urethral calculi patients in an endemic region and an undiagnosed primary fossa navicularis calculus. Urol Res. 2006; 34:37-40.

11. Ramello A, Vitale C, Marangella M. Epidemiology of nephrolithiasis. J Nephrol. 13(Suppl 3):S45-S50.

12. Lotan Y, Antonelli J, Buendia Jiménez I, Gharbi H, Herring R, Beaver A, and al. The kidney stone and increased water intake trial in steel workers: results from a pilot study. Published online: 26 Mai 2016 DOI 10.1007/s00240-016-0892-7.

13. Soucie J M, CoatesRJ, McClellan W, Austin H, and Thun M. Relation between geographic variability in kidney stone prevalence and risk factors for stones. Am J Epidemiol. 143: 487-495.

14. Kamal BA, Anikwe RM, Darawani H, et al. Urethral calculi: presentation and management. BJU Int. 2004; 93:549-552.

15. Jorgensen JB, Jensen KE Uroflowmetry. In: Boone TB (ed) The Urologic Clinics Of North America, Urodynamics, vol 1.WB Saunders, Philadelphia, p 239.

16. Larkin GL, Weber JE. Giant Urethral Calculus: A Rare cause of urinary retention. J Emerg Med 14(6):707709.
17. V. Singh y R.J. Sinha. Retención urinaria aguda en varones jóvenes y de mediana Edad debida a cálculos en la uretra: experiencia de un solo centro del norte de India. Actas Urol Esp. 2012;36(2):75-78.

18. A Bedii Salman. Urethral calculi in children. Journal of Pediatric Surgery, Vol31, No 10 (October), 1996: pp 1379-1382.

19. Ahmed S. Safwat, Diaa A. Hameed, Mohamed A. Elgammal, Yasser M. Abdelsalam, and Ahmad Abolyosr. Percutaneous Suprapubic Stone Extraction for Posterior Urethral stones in Children: Efficacy and Safety. UROLOGY 82: 448e450, 2013.

20. SHARFI AR. Presentation and Management of Urethral Calculi. British Journal of Urology (1991), 68, 211-212.

21. Diabate I, Sow I. Diverticule de l'urètre féminin contenant de multiples calculs. Prog Urol (2011) 21, $229-232$.

22. Thomas JS, Crew J. Obstructing urethral calculus in a woman revealed to be the cause of chronic pelvic pain. Int Urogynecol J (2012) 23:1473-1474.

23. AhmedA, Saeed NM. Experience with the management of urethral stones presenting with urinary retention at Gusau. Niger J Clin Pract. 2008 Dec; 11(4):309-11.

24. Hassan I, Mahammed I. Urethral calculi: a review. East Afr Med J. 1993 Aug; 70(8):523-5.

25. El-Sherif AE, El-Hafi R. Proposed new method for nonoperative treatment of urethral stones. J Urol 146:1546-1547.

26. Michael J. Ramdass and Vijay Naraynsingh. Multiple Urethral Stones Causing Penile Gangrene.Urology Volume 2014, Article ID 182094, 3 pages. 


\title{
Lithiasis Impacted in the Urethra: Pathology Not so Rare in Somine Dolo Hospital of Mopti.
}

\begin{abstract}
Introduction: Urethra is not a frequent localization of lithiasis. Many studies report clinical cases or limited series. The objective of this study was to show the frequency of this pathology in our practice.

Patients and Methods: It was retrospective descriptive study of the patients treated for urethral lithiasis between June 1, 2012 and June 30, 2016 in our structure.

Results: 106 cases of urethral lithiasis were diagnosed and treated. This localization accounted for 14, 54\% of the urolithiasis and $3,13 \%$ of urological intervention in the period. The mean age was 14, 24 years (1-68 years). The sex ratio was 7,15 in men favor. The reason for consultation was acute urinary retention in $66,98 \%$ of cases, the liathiasis was palpable in 83 , $02 \%$ of cases. The treatment consisted of a cystolithotomy after discharge of the lithiasis in the bladder in $74,53 \%$ of cases, instrumental extraction in $21,70 \%$ of cases and the urethrolithotomy in 3, 77\% $(n=4)$ of cases. The urethra was permeable after ablation of lithiasis in all cases.

Conclusion: The lithiasis impacted in the urethra is not rare in our practice. It must be evoked before acute urinary retention of children and adolescent.
\end{abstract}

Keywords: Lithiasis, urethra, acute urinary retention, cystolithotomy. 